\title{
Pemphigus Herpetiformis-Type Drug Reaction Caused by Programmed Cell Death Protein-I Inhibitor Treatment
}

\author{
Yunfang Zhang ${ }^{1, *}$ \\ Ming Zhang ${ }^{2, *}$ \\ Jianping $\mathrm{Xie}^{2}$ \\ Weiwei $\mathrm{Wu} \mathbb{( D}^{2}$ \\ Jiejie $\mathrm{Lu}^{2}$
}

'Department of Oncology, Hainan Provincial Hospital of TCM, Haikou, 570203, People's Republic of China;

${ }^{2}$ Department of Dermatology, The Fifth People's Hospital of Hainan Province, Branch of National Clinical Research Center for Skin and Immune Disease, Haikou, 570206, People's Republic of China

*These authors contributed equally to this work
Correspondence: Jiejie Lu

Department of Dermatology, The Fifth People's Hospital of Hainan Province, Branch of National Clinical Research Center for Skin and Immune Disease, No. 33, Southern Road of Longkun, Haikou, 570206, People's

Republic of China

Tel +86-135I 9816255

$\mathrm{Fax}+86-898-66729550$

Email lujiejie677@I63.com

\begin{abstract}
Reports of immune-related adverse events caused by programmed cell death protein-1 inhibitor are becoming increasingly frequent. Herein, we report the first case of pemphigus herpetiformis-type drug reaction presented after the treatment of tislelizumab (6 cycles) in a primary non-small cell lung carcinoma patient. A 56-year-old Chinese man was referred to our department for pruritic annulare erythema and blister for two weeks. Histological finding revealed blister formation in the epidermis and eosinophilic infiltration in the blister fluid. Direct immunofluorescence showed intercellular deposition of IgG and C3 within the lower part of epidermis. Serum anti-intercellular antibodies were positive at 1:100 dilution. Based on history and clinicopathological correlation, herpetiformis-type druginduced pemphigus was diagnosed, which was possibly be induced by tislelizumab. To the best to our knowledge, there is no report of pemphigus herpetiformis-type drug-induced reaction associated with programmed cell death protein-1 inhibitor treatment.
\end{abstract}

Keywords: programmed cell death protein-1 inhibitor, tislelizumab, pemphigus herpetiformis, drug reaction

\section{Introduction}

In recent years, immune checkpoint inhibitors including programmed cell death protein 1 (PD-1) and its ligand programmed death ligand 1 (PD-L1) antibody have been major breakthrough therapies in cancer. However, by activating the immune system, they may also cause unique immune-related adverse events (irAEs). Dermatologic adverse events (AEs) are the most frequently observed side reactions of immune-checkpoint inhibitor therapy, including nonspecific skin rashes, pruritus, vitiligo, lichen dermatitis, psoriasis, bullous pemphigus, sarcoidosis, alopecia, and severe drug eruption. ${ }^{1}$ Among various dermatological irAEs, autoimmune bullous diseases have been noted. ${ }^{2}$ Our case demonstrated the first case of pemphigus herpetiformis-type drug reaction, which may be induced by tislelizumab.

\section{Case Presentation}

In November 2020, a 56-year-old Chinese man was referred to our department for pruritic annular erythema and blisters on his head, face and upper limbs for two weeks. The patient complained that half a month ago, blisters appeared on the lower lip without any obvious inducement, which gradually aggravated and spread to the head, face and upper limbs. He was misdiagnosed as "herpes simplex" and "herpes zoster" 
in the local hospital, and was treated with valaciclovir orally and intravenous drip of acyclovir. However, the patient had no obvious improvement, and the blisters gradually expanded to the forearm, trunk, and scalp. The patient complained of itching without pain, fever and other symptoms. In February 2020, the patient was diagnosed as nonsmall-cell lung carcinoma (subtype: lung squamous cell carcinoma (LUSC)) with adrenal metastasis (cT3N1M1bIV A stage). Paclitaxel (albumin bound) and carboplatin regimen were given in the next three months. Despite repeated and multiple chemotherapy, the condition was still progressing, so the treatment regimen was adjusted. Then, gemcitabine and tislelizumab were administrated intravenously every 21 days as a cycle. The patient received 6 cycles in total from June 2020 to October 2020. The patient had no medical history of autoimmune disease.

On physical examination, multiple annular and herpetiform vesicles and bulla containing clear fluid with a range of $0.5-2 \mathrm{~cm}$ in diameter over erythematous background on the scalp, face, trunk, upper limbs, hands were observed (Figure 1A-C). Nikolsky sign was positive. A few crusts were present on the erythematous background. The nails and oral mucosa were not involved.

The blood routine test showed WBC $15.0 \times 10^{9} / \mathrm{L}$, neutrophil $12.0 \times 10^{9} / \mathrm{L}$, lymphocyte $1.5 \times 10^{9} / \mathrm{L}$. The erythrocyte sedimentation rate $(41 \mathrm{~mm} / \mathrm{h})$ and C-reactive protein $(73 \mathrm{mg} / \mathrm{L})$ were increased. The serum activity of creatine kinase (lactate dehydrogenase [LDH], $319 \mathrm{U} / \mathrm{L}$, and alphahydroxybutyrate dehydrogenase, $259 \mathrm{U} / \mathrm{L}$ ) was increased. No obvious abnormality was detected in routine tests of hepatic and renal function tests, blood sugar, lipid profile, and total IgE concentrations. Indirect immunofluorescence (IIF) assay revealed serum anti-intercellular antibodies was positive at 1:100 dilution and anti-basement membrane zone (BMZ) antibodies was negative. Histopathological findings of a bulla revealed epidermal spongiosis, intraepidermal cleft, acantholytic cells in the bulla, edema and mixed type inflammatory infiltration containing eosinophils in the papillary dermis (Figure $1 \mathrm{G}$ and $\mathrm{H}$ ). Direct immunofluorescence (DIF) assay of the perilesional skin showed intercellular intraepidermal $\mathrm{C} 3$ deposition and $\mathrm{IgG}$ deposition within the lower part of epidermis (Figure 1I and J).
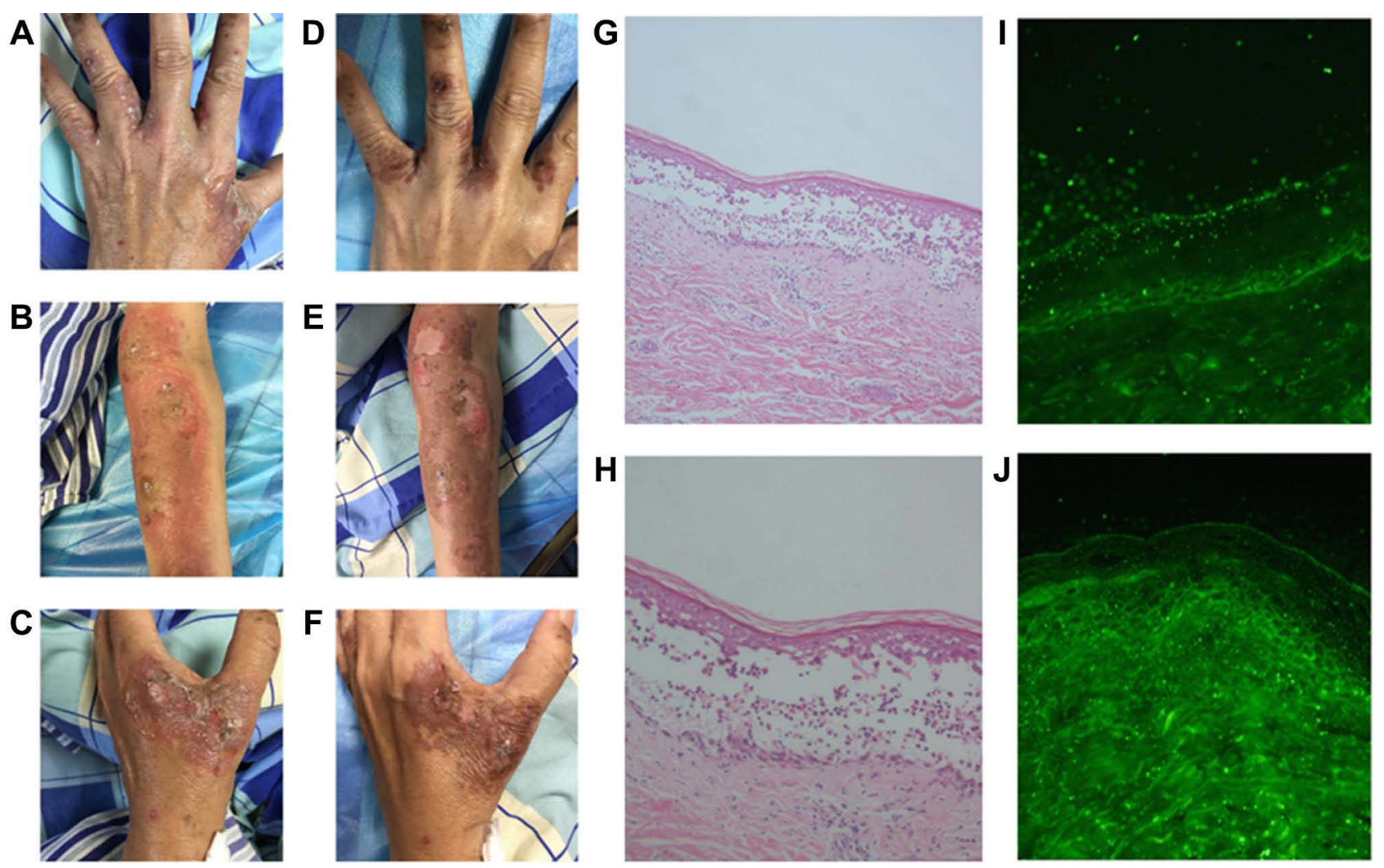

Figure I Clinical manifestation and histopathological findings of herpetiformis-type drug-induced pemphigus caused by tislelizumab before and after glucocorticoid therapy. (A-C) Clinical manifestation before glucocorticoid therapy; (D-F) clinical manifestation after glucocorticoid therapy; (G and $\mathbf{H})$ histopathological findings: epidermal spongiosis, intraepidermal cleft, acantholytic cells in the bulla, edema and mixed type inflammatory infiltration containing eosinophils in the papillary dermis (H\&E staining, G, $\times 100 ; \mathrm{H}, \times 200$ ); (I and J) direct immunofluorescence (DIF) findings: intercellular intraepidermal C3 deposition (I) and IgG deposition (J) within the lower part of epidermis. 


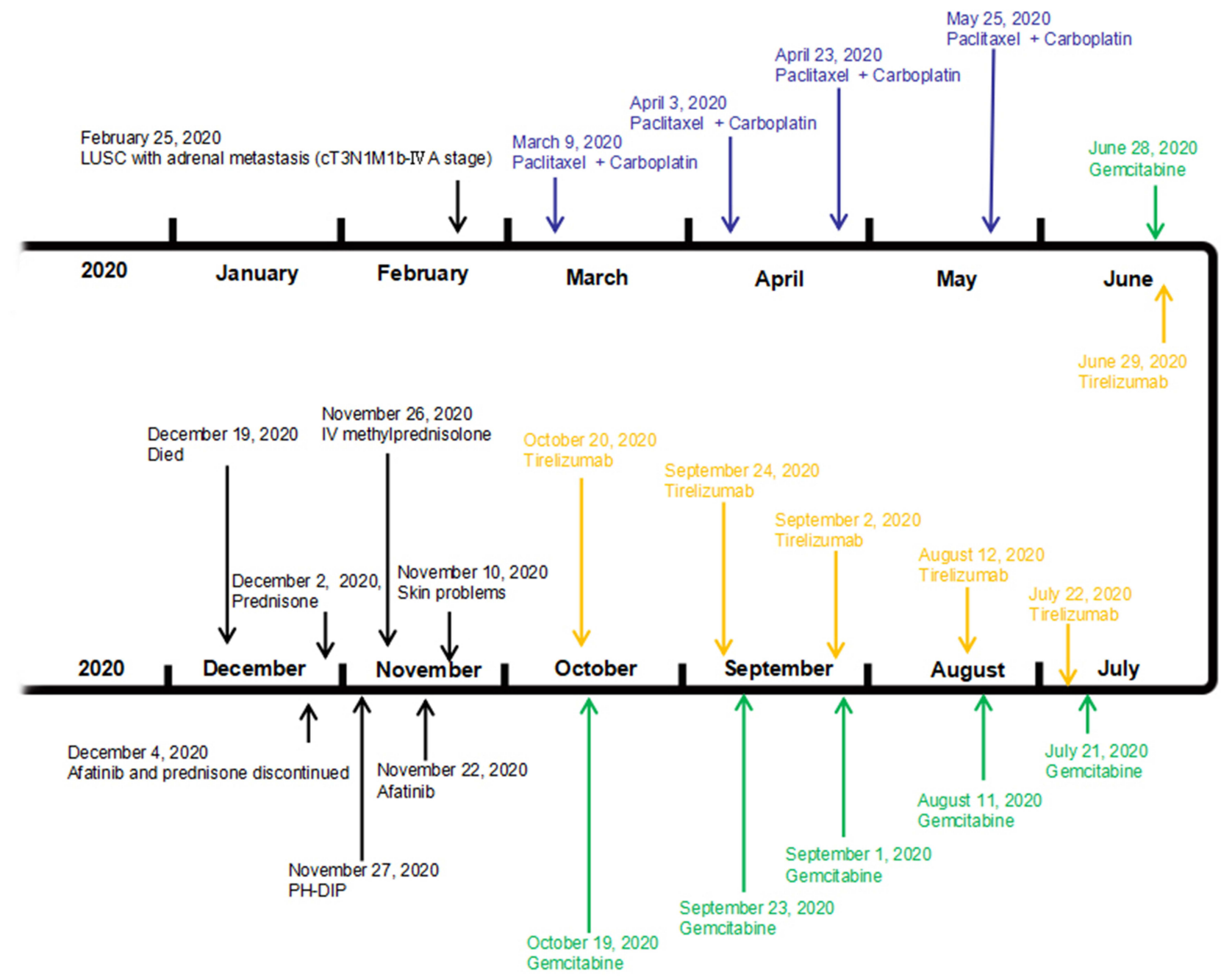

LUSC: lung squamous cell carcinoma

Paclitaxel+ Carboplatin: paclitaxel(albumin bound), $400 \mathrm{mg}, \mathrm{d} 1$, d8, d15 plus carboplatin $500 \mathrm{mg}, \mathrm{d} 1$

Gemcitabine: gemcitabine $1600 \mathrm{mg}, \mathrm{d} 1$, d8

Tirelizumab: tirelizumab $200 \mathrm{mg}, \mathrm{d} 1$

Afatinib: oral afatinib $40 \mathrm{mg} / \mathrm{d}$

PH-DIP: pemphigus herpetiformis type drug-induced penphigus

IV methylprednisolone: IV methylprednisolone $40 \mathrm{mg} /$ day

Prednisone: oal prednisone $40 \mathrm{mg} / \mathrm{d}$

Died: multiple organ failure as a consequence of airway obstruction

Figure 2 The timeline of the patient's diagnosis and treatments.

Depending on history and clinicopathological correlation, the patient was diagnosed as pemphigus herpetiformis $(\mathrm{PH})$ type drug-induced pemphigus (DIP), which may possibly be caused by tislelizumab. He was put on systemic therapy with IV methylprednisolone ( $40 \mathrm{mg} /$ day) for six days. The blisters and erythema improved (Figure 1D-F). Then, the patient continued to take oral prednisone $40 \mathrm{mg} / \mathrm{day}$. The blisters and erythemas improved, whereas his respiratory condition rapidly worsened owing to carcinoma progression. On December 19, 2020, the patient died of multiple organ failure as a consequence of airway obstruction. The timeline of the patient's diagnosis and treatments is presented in Figure 2.

The patient provided written informed permission to have any accompanying photos and case details published. The Hospital Ethics Committees of the Fifth People's Hospital of Hainan Province approved to publish the case details. 


\section{Discussion}

Herpetiform pemphigus (HP) is a rare variant of pemphigus, accounting for $6-7.3 \%$ of all pemphigus diseases. ${ }^{3}$ The cause of pemphigus herpetiformis has not been elucidated. There are reports of drug-induced pemphigus herpetiformis induced by erdosteine, D-penicillamine and bucillamine. ${ }^{4,5}$ According to the diagnostic criteria proposed by Costa et al. in $2019,{ }^{3}$ the patient's condition was consistent with pemphigus herpetiformis. Given that the patient had a history of lung squamous cell carcinoma, it should be differentiated from paraneoplastic pemphigus (PNP). Svoboda et al. proposed the revised diagnostic criteria for paraneoplastic pemphigus based on literature analysis, which requires three major criteria, or two major and both minor criteria: Major criteria, (a) Mucous membrane lesions with or without cutaneous involvement; (b) Concomitant internal neoplasm; (c) Evidence of antiplakin antibodies, including but not limited to IP, IB, ELISA, IIF on transitional epithelium. Minor criteria, (a) Acantholysis and/or lichenoid interface observed on histopathology, \pm necrotic keratinocytes; (b) DIF displaying intercellular and/or basement membrane staining. ${ }^{6}$ In the present case, the patient had no obvious oral mucosa involvement, erythema multiforme-like and lichen planus-like lesion. Histopathology did not show keratinocyte necrosis, liquefaction degeneration of basal cell, and dense band-like infiltration of lymphocytes in dermis. The patient was sensitive to glucocorticoid therapy. Although the patient had a history of lung squamous cell carcinoma, the patient did not conform to the diagnosis of paraneoplastic pemphigus.

As stated in the medical history, dermatologic problems started in mid-Nov 2020, at the end of gemcitabine and tislelizumab administration (June 2020-Oct 2020). Given the strict temporal relationship between onset of dermatological problems and gemcitabine and tislelizumab treatment, the above drugs were considered to be the probable cause. As an antimetabolite nucleoside chemotherapy, rash is reported to be the most common cutaneous adverse effect of gemcitabine. Other cutaneous adverse reactions that have been reported include bullous dermatosis, pseudocellulitis, subacute cutaneous lupus, alopecia, and palmar-plantar erythrodysesthesia. ${ }^{7}$ However, in our review of the available literature, autoimmune bullous diseases caused by gemcitabine have not been reported.
There were several reports that autoimmune bullous disease can be induced by immune checkpoint inhibitors, for example nivolumab, pembrolizumab and durvalumab. ${ }^{2,8,9}$ Tislelizumab is a programmed cell death receptor-1 (PD-1) monoclonal IgG4 antibody which is being developed as an immunotherapeutic, antineoplastic drug. Immune-related adverse reactions have been reported with treatment with tislelizumab, including hypothyroidism (7.4\%), skin adverse reactions $(6.6 \%)$, hyperthyroidism $(3.4 \%)$, pulmonary inflammation $(2.9 \%)$, hepatitis $(1.8 \%)$, diarrhea and colitis $(1.1 \%)$, other thyroid disorders $(0.6 \%)$, type 1 diabetes $(0.4 \%)$, adrenal insufficiency $(0.2 \%)$, myocarditis $(0.2 \%)$, nephritis $(0.2 \%)$ and pancreatitis $(0.1 \%) .{ }^{10}$ However, there is no report of pemphigus herpetiformis associated with programmed cell death protein-1 inhibitor.

\section{Conclusion}

We speculate that the present case of pemphigus herpetiformis-type drug reaction maybe induced by the treatment of tislelizumab, which was firstly reported.

\section{Ethics Statement}

The publications of images were included in the patient's consent for publication of the case.

\section{Consent Statement}

Informed consent was provided by the patient for publication of the case.

\section{Funding}

This work was supported by the Construction Project of Hainan Province Clinical Medical Center.

\section{Disclosure}

Yunfang Zhang and Ming Zhang are co-first authors for this study. The authors have no conflicts of interest to declare.

\section{References}

1. Han Y, Wang J, Xu B. Cutaneous adverse events associated with immune checkpoint blockade: a systematic review and meta-analysis. Crit Rev Oncol Hematol. 2021;163:103376. doi:10.1016/j. critrevonc.2021.103376

2. Naidoo J, Schindler K, Querfeld C, et al. Autoimmune bullous skin disorders with immune checkpoint inhibitors targeting PD-1 and PD-L1. Cancer Immunol Res. 2016;4(5):383-389. doi:10.1158/23266066.CIR-15-0123 
3. Costa LMC, Cappel MA, Keeling JH. Clinical, pathologic, and immunologic features of pemphigus herpetiformis: a literature review and proposed diagnostic criteria. Int $J$ Dermatol. 2019;58 (9):997-1007. doi:10.1111/ijd.14395

4. Yoshimura K, Ishii N, Hamada T, et al. Clinical and immunological profiles in 17 Japanese patients with drug-induced pemphigus studied at Kurume University. $\mathrm{Br} J$ Dermatol. 2014;171(3):544-553. doi:10.1111/bjd.12925

5. Akoglu G, Yavuz SO. Pemphigus herpetiformis-type drug reaction caused by erdosteine containing mucolytic in a child. Cutan Ocul Toxicol. 2017;36(3):302-304. doi:10.1080/15569527.2016.1254650

6. Svoboda SA, Huang S, Liu X, et al. Paraneoplastic pemphigus: revised diagnostic criteria based on literature analysis. J Cutan Pathol. 2021:1-6.doi: 10.1111/cup.14004
7. Haydock MM, Sigdel S, Pacioles T. Gemcitabine-induced skin necrosis. SAGE Open Med Case Rep. 2018;6:2050313X18809268. doi:10.1177/2050313X18809268

8. Krammer S, Krammer C, Salzer S, et al. Recurrence of Pemphigus Vulgaris Under Nivolumab Therapy. Front Med. 2019;6:262. doi:10.3389/fmed.2019.00262

9. Yatim A, Bohelay G, Grootenboer-Mignot S, et al. Paraneoplastic Pemphigus Revealed by Anti-programmed Death-1 Pembrolizumab Therapy for Cutaneous Squamous Cell Carcinoma Complicating Hidradenitis Suppurativa. Front Med. 2019;6:249. doi:10.3389/ fmed.2019.00249

10. BeiGene. Tislelizumab; China prescribing information [Chinese]; 2019. Available from: http://drugs.medlive.cn/drugref/html/21697. shtml. Accessed March, 102020.

Clinical, Cosmetic and Investigational Dermatology

\section{Publish your work in this journal}

Clinical, Cosmetic and Investigational Dermatology is an international, peer-reviewed, open access, online journal that focuses on the latest clinical and experimental research in all aspects of skin disease and cosmetic interventions. This journal is indexed on CAS.
The manuscript management system is completely online and includes a very quick and fair peer-review system, which is all easy to use. Visit http://www.dovepress.com/testimonials.php to read real quotes from published authors. 